\title{
Radar-Assisted UAV Detection and Identification Based on 5G in the Internet of Things
}

\author{
Jingcheng Zhao $\mathbb{D}^{D}$, Xinru Fu $(\mathbb{D}$, Zongkai Yang, and Fengtong Xu \\ School of Electronics and Information Engineering, Beihang University, China \\ Correspondence should be addressed to Xinru Fu; zy1702412@buaa.edu.cn
}

Received 4 April 2019; Revised 2 June 2019; Accepted 23 June 2019; Published 14 July 2019

Guest Editor: Bo Rong

Copyright ( 2019 Jingcheng Zhao et al. This is an open access article distributed under the Creative Commons Attribution License, which permits unrestricted use, distribution, and reproduction in any medium, provided the original work is properly cited.

\begin{abstract}
Unmanned aerial vehicles (UAVs) have broad application potential for the Internet of Things (IoT) due to their small size, low cost, and flexible control. At present, the main positioning method for UAVs is the use of GPS. However, GPS positioning may be affected by stronger electromagnetic signals from spoofing attacks. In this study, a radar-assisted positioning method based on $5 \mathrm{G}$ millimeter waves is proposed. In $5 \mathrm{G}$ end-to-end network slices, the rotors of UAVs can be detected and identified by deploying 5G millimeter wave radar. High-resolution range profile (HRRP) is used to obtain the UAV location in the detection zone. MicroDoppler characteristics are used to identify the UAVs and the cepstrum method is used to extract the number and speed information of the UAV rotor. The sinusoidal frequency modulation (SFM) parameter optimization method is used to separate multiple UAVs. The proposed method provides information on the number of UAVs, the position of the UAV, the number of rotors, and the rotation speed of each rotor. The simulation results show that the proposed radar detection method is well suited for UAV detection and identification and provides a valid GPS-independent method for UAV tracking.
\end{abstract}

\section{Introduction}

With the maturity of unmanned aerial vehicle (UAV) technology and the improvement of relevant laws and regulations, UAVs are increasingly being used for the development of the Internet of Things (IoT). For example, UAVs have been broadly used in the military IoT, smart agriculture, and smart cities to obtain and transmit geospatial information, sensor data information, and controlling information. In smart agriculture, UAV systems are used to gather near realtime remote sensing data for precision farming. In such applications, UAV systems need to be positioned precisely to obtain data from sensors. In smart cities, almost all aspects of the city are combined with the IoT, a task that requires large amounts of data transmission. This data volume requires a large number of base stations for IoT data transmission. Researchers have equipped UAVs with various sensors, such as high-definition cameras, as well as temperature, humidity, and air pollution sensors. In addition, todays UAVs are equipped with high quality wireless communication functions, including 5G, Wifi, Bluetooth, radio-frequency identification (RFID), and other communication means [1]. This type of equipment means that UAVs have a very important role in the IoT and can act as a mobile air base station, providing reliable downlink and uplink communications to terrestrial users, thereby increasing the capacity of wireless networks [2]. In addition, UAVs can be used as airborne wireless relays, as well as signal boosters and mobile nodes for the IoT and sensor networks [3]. If they are used as reliable air base stations, UAVs need to be positioned precisely. Another application of UAVs is for product delivery, in which case the UAVs location has to be monitored constantly. In all the aforementioned applications, the positioning of UAVs is very important. However, due to the mobility of UAVs, in certain cases, accurate positioning remains an unsolved problem. In particular, high-precision positioning is very important for remote sensing and automatic waypoint navigation of UAVs [4]. If the UAV cannot be accurately positioned, the UAV may collide with objects or other aircraft or may be deceived, captured, or interfered with.

At present, the positioning technology of UAVs is mainly achieved by using the GPS system but this method is sensitive to certain environments. Theoretically, the GPS system only requires three satellites to obtain positioning data but in 
practice, the UAV needs to communicate with at least 10 satellites to obtain a stable GPS system. When flying indoors, through tunnels, or dense construction areas, the GPS signal is not reliable and UAV positioning based on the GPS system will cause difficulties [5]. In addition, if UAVs are used for transporting goods, if criminals want to capture UAVs, it is difficult to prevent these attacks using GPS systems. Since the civilian GPS signal is not encrypted, the signal structure and data of UAV positioning system used in the civil IoT is predictable. This characteristic makes the civilian Global Navigation Satellite System (GNSS) signals a simple target for spoofing attacks. Moreover, after the UAV is captured, the criminal can take control of the captured UAV and fly away from its original flight path, while the original control source still receives data that appear correct and no alert is sent. This results in great risk in the use of UAVs. Many different technologies that assist in the positioning of UAVs have been developed, such as visual aids. However, visual aids are inevitably prone to drifting during long-range surveys without a priori feature maps and are only suitable for good weather and lighting conditions [6].

In this study, the precise positioning of UAVs is achieved through radar-assisted detection and identification of UAV technology. The method prevents many problems associated with traditional GPS positioning technology, such as GPS signal failure indoors, satellite occlusion areas, and interference with GPS signals. The radar-assisted detection and identification method has the advantage of working under all weather conditions and is capable of extracting additional features of the UAV due to the micro-Doppler features [7]. According to the current technology development plan, 5G will adopt the previous generations of wireless solutions ( $3 G$ and $4 G$ ) and introduce new promising mmWave technology so that a $5 \mathrm{G}$ base station not only can serve as a communication site for $5 \mathrm{G}$ networks but can also provide many powerful radar sensors in a functional device network, allowing for the detection of drones in urban environments [8-10]. The background of the UAV flight area is usually complex with other aircraft and birds; therefore, advanced clutter suppression and target detection algorithms are required. Following target detection, it is determined whether the detected target is a UAV and its category is identified. For the purposes of classification and identification, several features unique to UAVs are extracted [11]. In Doppler radar, the Doppler effect is used to determine the velocity of distant objects and accurate measurements can be obtained of the radial component of a targets velocity relative to the radar. Stepped frequency waveforms (SFW) are used to produce a high-resolution range profile (HRRP) of the target. With a wide-band Doppler radar, the HRRP and Doppler information of the target can be obtained to detect and identify UAVs.

The rest of this paper is organized as follows. The second part introduces the system model of the radar-assisted UAV positioning method in the IoT. In the third part, the scattering model of the UAV and rotors is established, the HRRP of a rotor-type UAV is introduced, and the micro-Doppler effect of the UAV is analyzed. The cepstrum method is used to extract the speed of the UAV and the number of rotors and the method for identifying multiple UAVs is

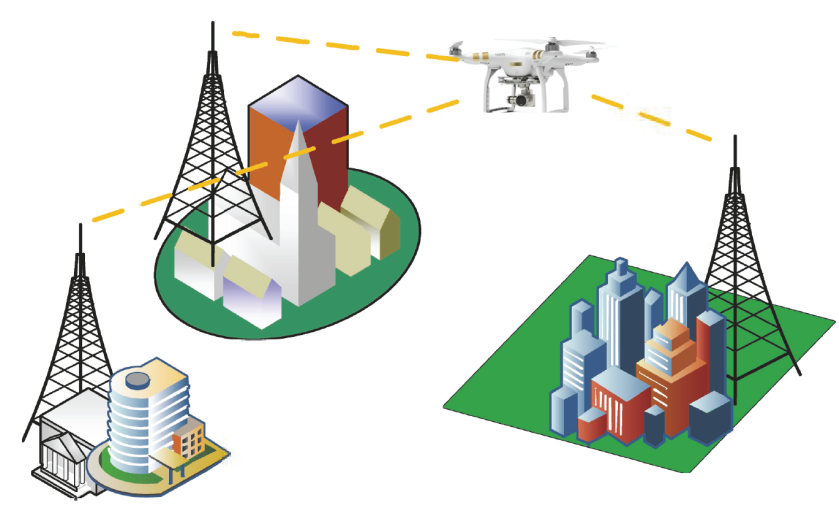

FIGURE 1: UAV detection based on 5G network.

described. In the fourth part, the simulation results and onedimensional imaging of a multitarget model of two UAVs are provided. The identification of the UAV based on the micro-Doppler information is provided and the method for extracting the number and speed of the rotors are described. The conclusions are provided in the final section.

\section{System Model}

5G mmWave technology and distributed networks will facilitate radar detection and identification of small UAVs. The narrow steerable beam enables the base station to function as a millimeter wave radar system so that the base station is able to detect and identify drones in urban environments [12, 13]. In addition, through end-to-end fragmentation technology, the $5 \mathrm{G}$ network provides a logically isolated virtual private segmentation network for different services to implement a manual flight-specific traffic channel network [14].

The model for detecting and identifying the UAV in the $5 \mathrm{G}$ network is shown in Figure 1. The detection network channel of the UAV is established in the 5G network and the millimeter wave base station is established. The electromagnetic signal is transmitted by the millimeter wave base station and beamforming technology is used to reduce the interference of the clutter signal $[15,16]$. The reflected echo of the target is obtained by multipath reflection, and the location of UAV is transmitted through the data processing center $[17,18]$. Accurate identification and positioning are achieved in a timely manner.

2.1. Radar-Assisted Detection and Identification Method. The flowchart of the process of radar-assisted detection and identification of UAVs is shown in Figure 2. The $5 \mathrm{G}$ millimeter wave radar transmits a wideband signal to the target area and receives the echo reflected from the target. The HRRP provides the number of targets in the detection area. For a single target, the Doppler method is used to determine whether the target is a UAV and the cepstrum is used to extract the rotor parameters of the UAV. For multiple targets, after the Doppler and cepstrum analyses, the sinusoidal frequency modulation (SFM) parameter optimization method is used to obtain the 


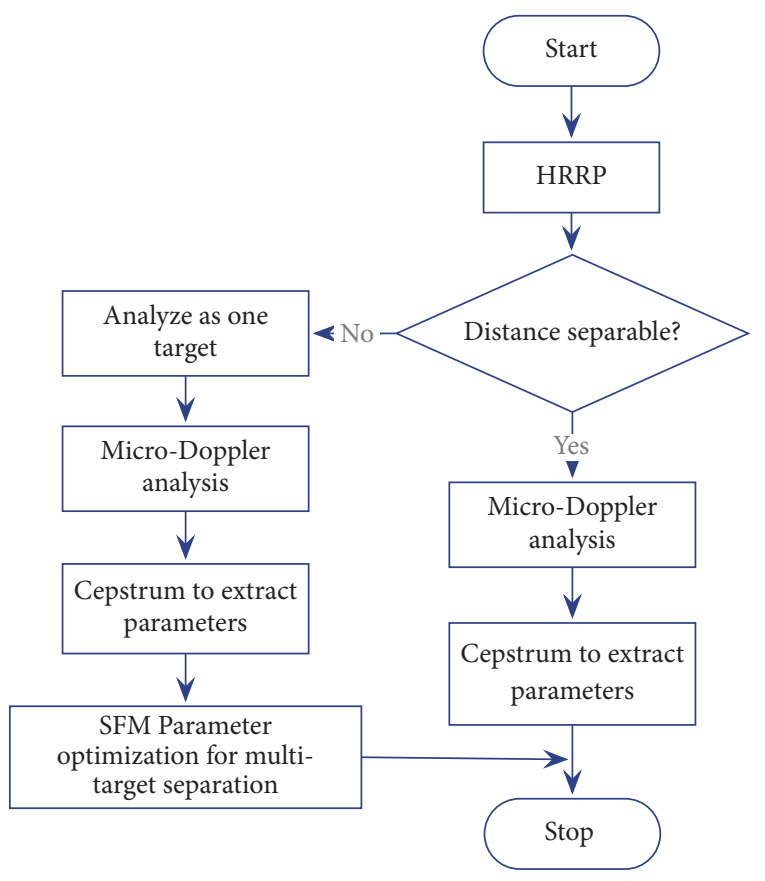

FIGURE 2: Flowchart of radar-based identification.

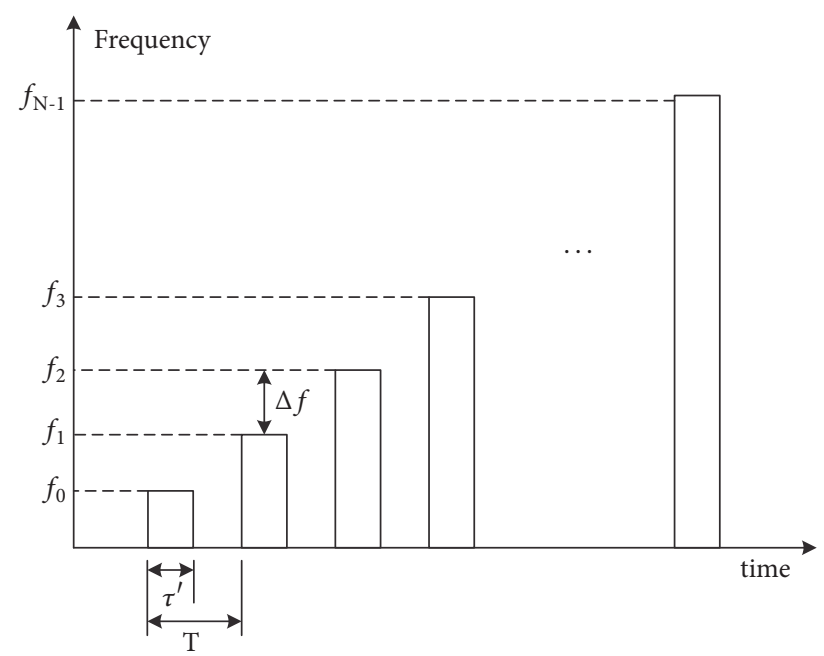

FIGURE 3: SFW burst.

position of each rotor so that multiple targets can be separated and identified.

2.2. Stepped Frequency Waveforms. High bandwidth, low latency, and good beamforming of $5 \mathrm{G}$ networks provide a reliable basis for resolution because future $5 \mathrm{G}$ mmWave systems cannot only support very high carrier frequencies but also wider bandwidths (effective operation up to $2 \mathrm{GHz}$ ). Wider bandwidth facilitates the detection of UAVs flying in close formation. The SFW is a type of $5 \mathrm{G}$ mmWave signal and produces an HRRP of the target. A typical SFW burst is shown in Figure 3; it consists of N narrow-band pulses. The frequency from pulse to pulse is stepped by a fixed frequency and each group of pulses is called a pulse train [19].
Within a pulse width $\tau^{\prime}$, the transmitted signal of the nth pulse is defined in

$$
s(n, t)=\rho_{n} \cos \left(2 \pi f_{n} t+\theta_{n}\right), \quad n T \leq t \leq n T+\tau^{\prime}
$$

where $\theta_{n}$ is the initial phase of the pulse and $\rho_{n}$ is the amplitude constant.

The echo signal of the target is defined in

$$
s_{r}(n, t)=\rho_{n}^{\prime} \cos \left(2 \pi f_{n}(t-\tau(t))+\theta_{n}\right)
$$

where $\rho_{n}^{\prime}$ is the amplitude of the echo signal and $\tau(t)$ is the echo delay of the signal; $\tau(t)$ is expressed as

$$
\tau(t)=\frac{2 R(t)}{c}
$$

where $c$ is the speed of light and $R(t)$ is the distance between the target and the radar at time $t$.

The received signal is split in the quadrature phase detector and enters the mixer of the two synchronous detectors. In the first mixer, the reference signal and the received signal are mixed and the $90^{\circ}$ phase-shifted echo signal is mixed with the reference signal in the second mixer. After combining the outputs of the two mixers and low pass filtering, the complex signal is

$$
s_{r}(n, t)=\rho_{n} \exp \left\{-\mathrm{j}\left[2 \pi\left(f_{0}+n \Delta f\right) \frac{2 R(t)}{c}\right]\right\}
$$

If we assume that the targets' moving distance is less than one range resolution cell for each group of pulse signals, then $s_{r}(n, t)$ is expressed as

$$
s_{r}(n)=\rho_{n} \exp \left\{-\mathrm{j}\left[2 \pi\left(f_{0}+n \Delta f\right) \frac{2 R}{c}\right]\right\}
$$

The inverse discrete Fourier transform (IDFT) of $s_{r}(n)$ is

$$
s(k)=\frac{1}{N} \sum_{n=0}^{N-1} s_{r}(n) \exp \left(j \frac{2 \pi k n}{N}\right)
$$

By normalizing [19], (6) yields

$$
s(k)=\sum_{n=0}^{N-1} \exp \left[j \frac{2 \pi n}{N}\left(k-\frac{2 R \Delta f}{c}\right)\right]
$$

which can be simplified as

$$
s(k)=\frac{\sin \pi \chi}{\sin (\pi \chi / N)} \exp \left(j \frac{N-1}{2} \frac{\pi \chi}{N}\right)
$$

where $\chi=-2 N R \Delta f / c+k$. The peak of $|s(k)|$ appears at $k=2 N \Delta f R_{0} / c \pm m N, m \in Z$. The distance resolution is $c /(2 \mathrm{BW})$, where $\mathrm{BW}$ is the signal bandwidth.

2.3. Micro-Doppler Effect in Radar. In traditional radar applications, the antenna illuminates the target with a microwave signal and receives the echo reflected by the target. The echo signal includes the target characteristics of interest. For example, if the transmit signal hits a moving target, 
the carrier frequency of the echo signal shifts, which is called the Doppler effect. The Doppler shift reflects the moving speed of the target. Mechanical vibration or rotation of a structure on the target can also cause additional frequency shifts in the returned radar signal; this is known as the micro-Doppler effect. The micro-Doppler effect allows us to determine additional properties of the target [20].

In many cases, some components of the target may have rotations or vibrations in addition to the main target translation, such as a rotor on a UAV or the flapping wing of a bird. The motion dynamics of the rotating rotor or wing will frequency-modulate the backscattered signals and cause additional Doppler shifts near the center of the translational Doppler shift, which is known as the micro-Doppler shift. Therefore, the micro-Doppler shift can be considered a unique signature of targets with vibrations, rotations, or other nonuniform motions. The micro-Doppler shift depends on the signal wavelength, the vibration or rotation speed of the target, and the incident angle of the radar line of sight (LOS) relative to the rotation or vibration plane. The reflected radar signal has the largest micro-Doppler shift when the radar LOS is parallel to the rotation or vibration plane. When the radar LOS is perpendicular to the rotation or vibration plane, the reflected signal has the smallest micro-Doppler shift. The micro-Doppler shift has been widely used to classify targets with rotations or vibrations.

Almost all UAVs have at least one or more rotating rotors. For example, a four-rotor UAV has two rotors that rotate clockwise and two that rotate counterclockwise about a vertical axis. The UAV can take off vertically, hover, and fly forward, backward, and sideways. Other multirotor UAVs, such as UAVs with six or eight rotors, have matching rotor sets that rotate in opposite directions. Therefore, it is possible to detect and identify UAVs by using the microDoppler effect of the rotors. In this study, we extract the characteristics of UAVs based on the micro-Doppler effect [21].

\section{Detection and Identification Method of UAV}

3.1. Scattering Model of UAV with Rotors. A UAV is a slow small target that can be simplified to a point target in traditional radar; however, since we plan to identify the UAV by using information on the rotors, we have to develop a model of a UAV in which the rotor is separate from the fuselage. Figure 4 shows the scattering model of a rotor-type UAV. The distance between the center of the rotor and the radar is $R_{0}$ and the elevation angle of the radar observation is $\beta$.

The fuselage scattering of the UAV can be obtained by (4). For a rotating point with a center of rotation of $l$ and a rotational speed of $\Omega$, the position relative to the radar at time $t$ can be expressed as

$$
R(t)=R_{0}+l \cos \beta \cos \left(\Omega t+\varphi_{0}\right)
$$

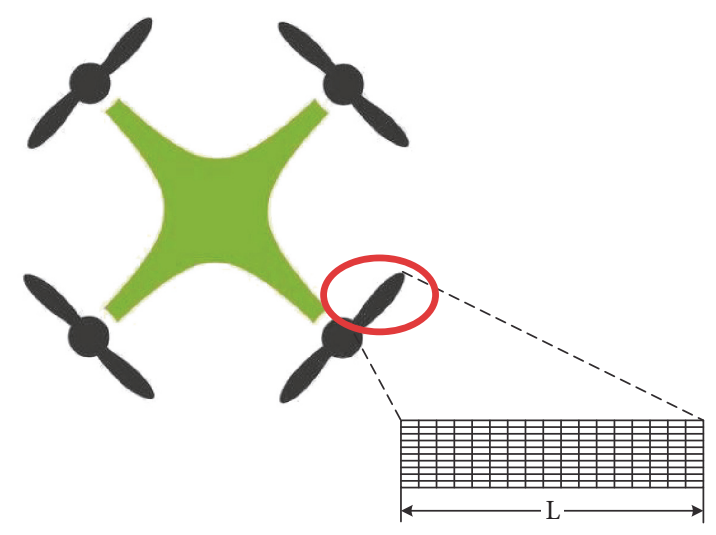

FIgURE 4: Scattering model of a rotor-type UAV.

Then the echo signal can be defined as

$$
\begin{array}{r}
s_{r}(n, t)=\rho \exp \left\{-\mathrm{j}\left[2 \pi\left(f_{0}+n \Delta f\right)\right.\right. \\
\left.\left.\times \frac{2\left(R_{0}+l \cos \beta \cos \left(\Omega t+\varphi_{0}\right)\right)}{c}\right]\right\}
\end{array}
$$

We assume that the blade length is $\mathrm{L}$ as shown in Figure 3. Each blade can be thought of as a uniform, rigid rectangular plate that consists of arrays of rectangular facets with the scattering center of the surface of each facet assigned to its tip. The baseband signal originating from the rotors of a UAV can be expressed as

$$
\begin{gathered}
s_{r}(n, t)=\sum_{i=1}^{N_{T}} \sum_{m=1}^{N_{M}} \sum_{k=1}^{N_{k}} \rho_{i, m, k} \exp \left(-\mathrm{j} \frac{4 \pi\left(f_{0}+\mathrm{n} \Delta f\right)}{c}\right. \\
\left.\times\left[R_{0 i, k}+l_{i, m, k} \cos \beta_{i, k} \cos \left(\Omega_{i} t+\varphi_{0 i, m}\right)\right]\right)
\end{gathered}
$$

where $N_{T}$ is the total number of rotors, $N_{M}$ is the number of blades per rotor, and $N_{k}$ represents the number of facets.

3.2. HRRP of Rotor-Type UAV. The movement of the UAV can be divided into two parts, namely, the movement of the main body of the UAV and the rotation of the rotor. Since the time of a one-step sweep measurement is about hundreds of milliseconds and the UAV is a small slow target, its displacement during the one-step sweep measurement is negligible. Therefore, the UAV can be regarded as a stationary target during the one-step sweep measuring time. In this study, we do not consider the movement of the fuselage.

$$
\begin{gathered}
s_{r}(t)=\sum_{i=1}^{N_{T}} \sum_{m=1}^{N_{M}} \sum_{k=1}^{N_{k}} \rho_{i, m, k} \exp \left(-\mathrm{j} \frac{4 \pi\left(f_{0}+\mathrm{PRF} \Delta f t\right)}{c}\right. \\
\left.\times\left[R_{0 i, k}+l_{i, m, k} \cos \beta_{i, k} \cos \left(\Omega_{i} t+\varphi_{0 i, k}\right)\right]\right)
\end{gathered}
$$


It is evident from (12) that the echo signal form of the rotor is an SFM signal and the equation can be expanded according to the following Bessel function:

$$
\mathrm{e}^{\mathrm{j} A \sin \theta}=\sum_{n=-\infty}^{\infty} \mathrm{J}_{n}(A) \mathrm{e}^{\mathrm{j} n \theta}
$$

where $J_{n}(\cdot)$ is the first type of an $\mathrm{n}$-order Bessel function.

The IDFT of the expanded rotor echo signal based on Eq. (13) can be obtained as [22]:

$$
\begin{aligned}
S_{r} & (f) \\
& =\sum_{i=1}^{N_{T}} \sum_{m=1}^{N_{M}} \sum_{k=1}^{N_{k}} \sum_{n=-N_{1}}^{N_{1}} b_{i, m, k, n} \times \delta\left(f-f_{R, i}-N_{M} n f_{\Omega, i}\right)
\end{aligned}
$$

where $f_{R, i}$ is the frequency offset caused by the position of the rotor center, $f_{\Omega, i}$ is the micro-Doppler frequency caused by the rotor speed, and $b$ is the Bessel coefficient. The signal obtained by (14) is a superposition of the pulse signals of different amplitudes with a period of $N_{m} f_{\Omega}$, which is a comblike function whose amplitude is modulated.

According to the characteristics of the Bessel function:

$$
\sum_{m=-\infty}^{\infty} J_{m}^{2}(A)=1
$$

The energy of the frequency-domain signal produces a divergence effect. The magnitude of the harmonic amplitude is modulated by the Bessel function. According to the Carson criterion [23], the number of harmonics is

$$
N_{1} \approx 2|A|+3
$$

The width of spectral broadening caused by the rotation of the rotor is [22]

$$
B \approx \frac{8 \pi f_{\Omega} L \cos \beta}{N_{M} \lambda_{0}}
$$

The rotational motion appears in the HRRP as a symmetrical range distribution centered on the position of the fuselage. When the rotor scattering is smaller than the fuselage scattering or slightly larger than the fuselage scattering, the amplitude of the rotor in the HRRP is much smaller than the amplitude of the fuselage because the rotor rotational motion is dispersed after the Bessel series expansion. For the purpose of detection, the influence of the rotor rotation on the HRRP can be neglected and the position information of the UAV can be obtained by directly reading the position of the main scattering from the fuselage. In most cases, the fuselage scattering is greater than the rotor scattering and the effects of the rotor rotation do not have to be considered for obtaining the UAV position.

When the rotor scattering is much larger than the fuselage scattering, the main body scattering is submerged in the distance harmonic caused by the rotation of the rotor. In this case, the harmonic boundary caused by the rotation can be estimated and the center position of the two boundary positions can be used to estimate the target position. Alternatively, the SFM parameter optimization method described below can be used to compensate for the effect of rotor rotation on the HRRP.

\subsection{UAV Identification}

3.3.1. Micro-Doppler Effect of UAV with Rotors. The Doppler shift of the received signal can be expressed as

$$
\begin{aligned}
f_{D} & =\frac{1}{2 \pi} \frac{\mathrm{d} \varphi(t)}{\mathrm{d} t}=\frac{2}{c}\left\{\left[f_{0}+P R F\right.\right. \\
& \times \Delta f t][-\Omega L \cos \beta \sin (\Omega t \\
& \left.\left.\left.+\varphi_{0}\right)\right]+\Delta f\left(L \cos \beta \cos \left(\Omega t+\varphi_{0}\right)+R_{0}\right) \times P R F\right\} \\
& =\frac{2}{c}\left\{L \cos \beta \sqrt{\left(\Omega f_{0}+\Omega P R F \times \Delta f t\right)^{2}+(\Delta f \times P R F)^{2}}\right. \\
& \cdot \cos (\Omega t+\theta)+\Delta f R_{0} \times P R F
\end{aligned}
$$

The micro-Doppler frequency shift changes over time and the joint time-domain analysis method is the most intuitive means to observe the target's micro-Doppler effect. Shorttime Fourier transform (STFT) [24] is a commonly used joint time domain analysis methods. The basic process of the STFT is to decompose a long time signal into shorter segments of the same length, calculate the Fourier transform on each short segment, and visualize the variation of the echo frequency over time.

3.3.2. Micro-Doppler Parameter Extraction Algorithm. The extraction of the parameters of the rotor, such as the number of rotors and the speed, is used to identify the type of UAV. For single-rotor UAVs, the STFT can be used for the identification. For multirotor UAVs, the micro-Doppler frequencies of different rotors are aliased together, which makes the target difficult to resolve and requires a new extraction method.

It is evident in (14) that the echo signals are superimposed on the pulse signals of different amplitudes in the frequency domain with $N_{m} f_{\Omega}$ as the period. The period of the signal is related to the rotor speed and the rotors of different speeds produce comb-like functions of different periods. The number of rotors can be determined by extracting the number of different periodic signals and the rotational speed of each rotor of the UAV is estimated by extracting the period of the signal.

Cepstrum is an effective method used in speech signal processing $[25,26]$ for the periodic detection of the pitch and is now also commonly used for mechanical state detection and fault diagnosis. In this study, the power cepstrum is used for parameter extraction. By multiplying the signal by its own conjugate signal, the value of the micro-Doppler can be doubled [11], which contributes to providing better periodic signal extraction. We set $g(t)$ to

$$
g(t)=s(t) * s^{*}(-t)
$$

A Fourier transform is performed on $g(t)$ and the result is expressed as $G(f)$. $G(f)$ can be regarded as a comb function $C(f)$ that is multiplied by some form of amplitude modulation $A(f)$. The results of $G(f)$ are shown in Figure 5. 


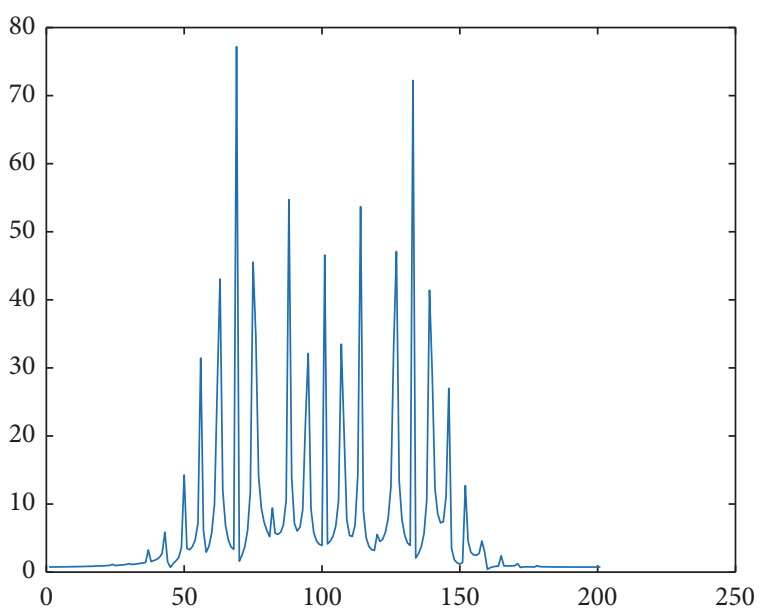

Figure 5: Function diagram of $G(f)$.

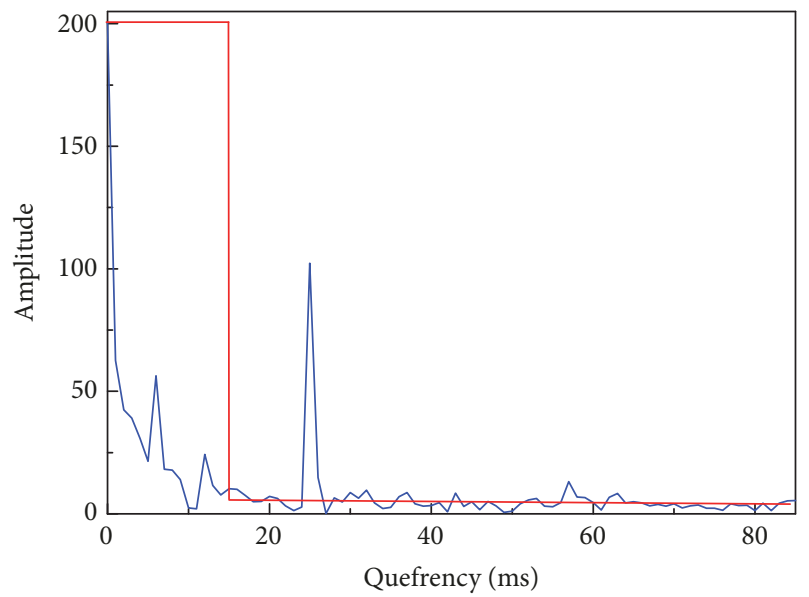

Figure 6: Cepstrum domain.

By calculating the log spectrum, the multiplicative relationship between the comb function and amplitude modulation is converted into a summation. Then, the inverse Fourier transform is performed and the square of the amplitude is used to obtain the cepstrum of the function.

The specific equation is as follows:

$$
\mathrm{C}(q)=|\operatorname{IFFT}\{\log |\operatorname{FFT}(g(t))|\}|^{2}
$$

The cepstrum domain is shown in Figure 6; the modulated function $A(f)$ is a nonperiodic function whose peak appears at the zero value of the cepstrum and $G(f)$ appears as a peak at its periodic frequency. By using a high-pass filter, the influence of $G(f)$ can be removed and the rotation period of the UAV is obtained.

3.4. Estimation of Multi-UAV Positions. The rotor rotation widens the HRRP distance. If the distance between two targets is large enough so that they can be separated in the HRRP, each target can be separately processed to determine whether it is a UAV. If the distance between the targets is very small due to distance expansion by rotation, a multi-SFM
TABLE 1: Variable simplification.

\begin{tabular}{lc}
\hline Initial parameter & Simplified parameters \\
\hline$\triangle f \frac{2 R_{0}}{c}$ & $f_{c}$ \\
\hline$\left(f_{0}+n \triangle f\right) \frac{2 l \cos \beta \cos \left(\Omega n / P R F+\varphi_{0}\right)}{c}$ & $m_{f i}$ \\
\hline$f_{0} \frac{2 R_{0}}{c}$ & $\theta$ \\
\hline$\frac{\Omega}{P R F}$ & $f_{m i}$ \\
\hline
\end{tabular}

signal separation method based on parameter optimization is used to compensate for the rotational motion.

3.4.1. Multi-SFM Signal Separation Based on Parameter Optimization. We discretize (12):

$$
\begin{array}{r}
s_{r}(n)=\sum_{i=1}^{N_{T}} \sum_{m=1}^{N_{M}} \sum_{k=1}^{N_{k}} \rho_{i, m, k} \exp \left(-\mathrm{j} \frac{4 \pi\left(f_{0}+\mathrm{n} \Delta f\right)}{c}\right. \\
\left.\times\left[R_{0 i, k}+l_{i, m, k} \cos \beta_{i, k} \cos \left(\frac{\Omega_{i} n}{P R F}+\varphi_{0 i, k}\right)\right]\right)
\end{array}
$$

Table 1 shows the simplified variables in (21): SFMs:

Equation (21) can be expressed in the form of multiple $x(n)$

$$
=\sum_{i=1}^{M} A_{i} \exp \left\{-j\left[2 \pi\left(f_{c i} n+m_{f i} \cos \left(2 \pi f_{m i} n+\varphi_{i}\right)+\theta_{i}\right)\right]\right\}
$$

The unknown parameters in (22) include the center frequency $f_{c i}$, frequency modulation factor $m_{f i}$, modulation period $f_{m i}$, initial phase of the SFM signal $\phi_{i}$, initial phase of the FM signal $\theta_{i}$, and the amplitude $A_{i} . f_{c i}$ is related to the target position and thus we mainly focus on the estimation of $f_{c i}$ from different signals. The parameter optimization method estimates the parameter estimation order of $f_{c i}$.

The modulation period of the signal can be obtained by the cepstrum method and will not be described here.

(a) Estimation of the modulation period $f_{m i}$.

(b) Estimation of the initial phase $\varphi_{i}$ and the frequency modulation factor $m_{f i}$.

We assume $\varphi_{i} \epsilon[0,2 \pi], m_{f i} \epsilon[m 1, m 2]$. The initial estimated value $\varphi_{i}=0, m_{f i}=m 1$. The reference signal can be expressed as

$$
\tilde{x}_{m i, \varphi i}(n)=\exp \left\{j\left[2 \pi \widehat{m}_{f i} \cos \left(2 \pi \widehat{f}_{m i} n+\widehat{\varphi}_{i}\right)\right]\right\}
$$

Equation (23) is multiplied by (22):

$$
\begin{aligned}
& x(n) \tilde{x}_{m, \varphi}(n)=\sum_{i=1}^{M} A_{i} \\
& \quad \cdot \exp \left\{-j\left[2 \pi\left(f_{c i} n+m_{f i} \cos \left(2 \pi f_{m i} n+\varphi_{i}\right)\right)\right]+\theta_{i}\right. \\
& \left.\quad+j\left[2 \pi \widehat{m}_{f i} \cos \left(2 \pi \widehat{f}_{m i} n+\widehat{\varphi}_{i}\right)\right]\right\}
\end{aligned}
$$


For the ith SFM component,

$$
\begin{aligned}
& x_{i}(n) \tilde{x}_{m i, \varphi i}(n)=A_{i} \\
& \cdot \exp \left\{-j\left[2 \pi f_{c i} n\left(+m_{f i} \cos \left(2 \pi f_{m i} n+\varphi_{i}\right)+\theta_{i}\right)\right]\right. \\
& \left.+j\left[2 \pi \widehat{m}_{f i} \cos \left(2 \pi \widehat{f}_{m i} n+\widehat{\varphi}_{i}\right)\right]\right\}=A_{i} \\
& \cdot \exp \left\{-j\left(2 \pi f_{c i} n\right)+\theta_{i}\right\} \exp \left\{-j 2 \pi\left[-2 m_{f i}\right.\right. \\
& \cdot \sin \left(2 \pi f_{m i} n+\frac{\varphi_{i}+\widehat{\varphi}_{i}}{2} \sin \left(\frac{\varphi_{i}-\widehat{\varphi}_{i}}{2}\right)\right) \\
& \left.\left.-\left(\widehat{m}_{f i}-m_{f i}\right) \cos \left(2 \pi \widehat{f}_{m i} n+\widehat{\varphi}_{i}\right)\right]\right\}
\end{aligned}
$$

(c) Estimation of $\widehat{f}_{c i}$ : when $\varphi_{i}=\widehat{\varphi}_{i}$, the spectrum of the signal is concentrated in a band of width $2\left|\widehat{m}_{f i}-m_{f i}\right| \widehat{f}_{m i}$. When $\widehat{m}_{f i}=m_{f i}$, the rotational motion is compensated. The original signal becomes

$$
A_{i} \exp \left\{-j\left(2 \pi f_{c i} n\right)+\theta_{i}\right\}
$$

Fast Fourier transform (FFT) is performed on (26); the position corresponding to the maximum value after the transformation is $\widehat{f}_{c i}$, which can be expressed as

$$
\widehat{f}_{c i}=\underset{f}{\arg \max }\left|F F T\left(x_{i}(n) \tilde{x}_{m i, \varphi i}(n)\right)\right|
$$

3.4.2. Rotor Position Estimation. The steps for identifying close-range multi-UAVs using the SFM parameter optimization method are as follows:

(a) The cepstrum method is used to calculate the rotation period of each rotating target.

(b) Since the fuselage scattering will affect the SFM parameter optimization method, the influence of the subject scattering must be removed prior to using the SFM parameter optimization method. First, two subsequent frames of signals are measured and subtracted from each other for the cancellation of the fuselage scattering. Second, HRRP is used to determine the maximum value of the rotor scattering. Finally, the maximum value is set as a threshold. If the single frame in the HRRP is larger than the threshold, the single signal is set as the mean value of the subtracted HRRP.

(c) The SFM parameter optimization method is used for the rotation periods of the rotors obtained in step (a) to determine their position.

\section{Simulation}

4.1. Simulation Model. Figure 7 shows a schematic of the simulation model. There are three targets in the observation area, i.e., a quad-rotor UAV at a distance of $300 \mathrm{~m}$ from the radar, a non-UAV target $800 \mathrm{~m}$ from the radar, and a singlerotor UAV $850 \mathrm{~m}$ from the radar. The rotor length of the quadrotor UAV is $0.12 \mathrm{~m}$ and the rotational speeds are $158 \mathrm{r} / \mathrm{s}, 150$ r/s, $142 \mathrm{r} / \mathrm{s}$, and $139 \mathrm{r} / \mathrm{s}$, respectively. The rotor length of the

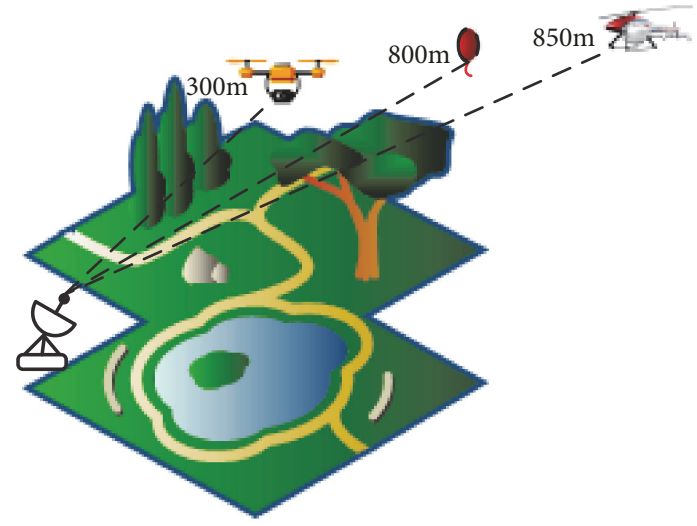

FIGURE 7: Schematic of the simulation model.

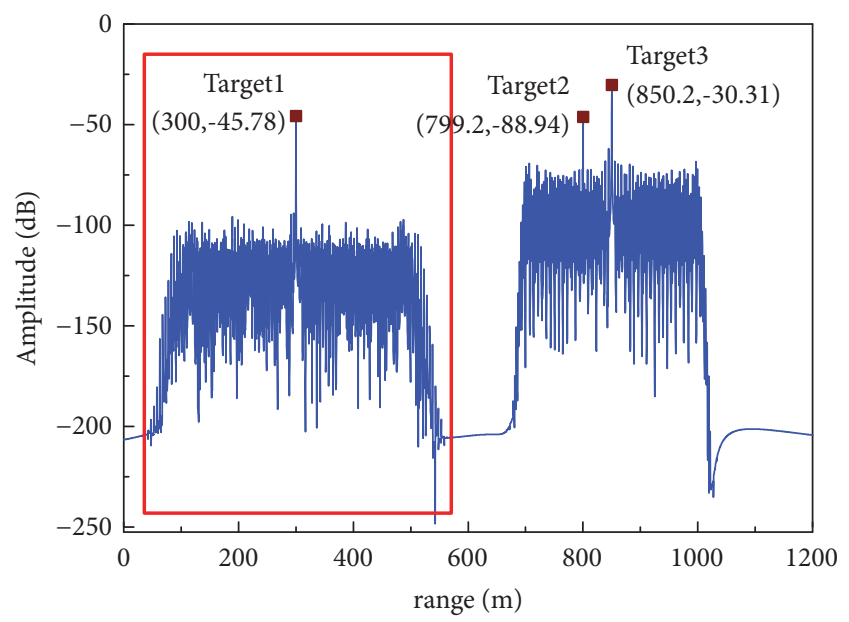

FiguRE 8: HRRP of the target.

single-rotor UAV is $0.15 \mathrm{~m}$ and the rotational speed is $88 \mathrm{r} / \mathrm{s}$. The parameters of the radar are as follows: starting frequency $F_{0}=25 \mathrm{GHz}$, which is in the $5 \mathrm{G}$ band; signal bandwidth BW $=500 \mathrm{MHz}$, the resolution cell is $0.3 \mathrm{~m}$; sampling points $\mathrm{N}=$ 8001; PRF=150 kHz.

4.2. Simulation Results. The HRRP of the echo signal is shown in Figure 8. Three targets are detected at distances of $300 \mathrm{~m}, 799.2 \mathrm{~m}$, and $850.2 \mathrm{~m}$ from the radar. Target 2 and target 3 cannot be separated in the HRRP because they are in close proximity and have to be analyzed as one target. The SFM optimization method can be used to determine the UAV locations. Target 1 is far from target 2 and target 3 . Therefore, the time-domain echo signal of target 1 can be separated and identified by using a distance-domain window and FFT.

4.2.1. Identification of Target 1. Figure 9 shows the result of the micro-Doppler characteristics of target 1 . It can be inferred that target 1 is a rotor-type UAV. Due to the presence of the stepping frequency, the echo signal appears as a fixed frequency value that does not change over time in the timefrequency image. The micro-Doppler frequency caused by 
TABLE 2: Target parameters.

\begin{tabular}{lccc}
\hline & Target type & Position $(\mathrm{m})$ & Rotor speed $(\mathrm{r} / \mathrm{s})$ \\
\hline Target 1 & Quad-rotor UAV & 300 & $157.9,150.0,142.0,138.9$ \\
\hline Target 2 & Other targets & 799.2 & - \\
\hline Target 3 & One-rotor UAV & 850.2 & 88 \\
\hline
\end{tabular}

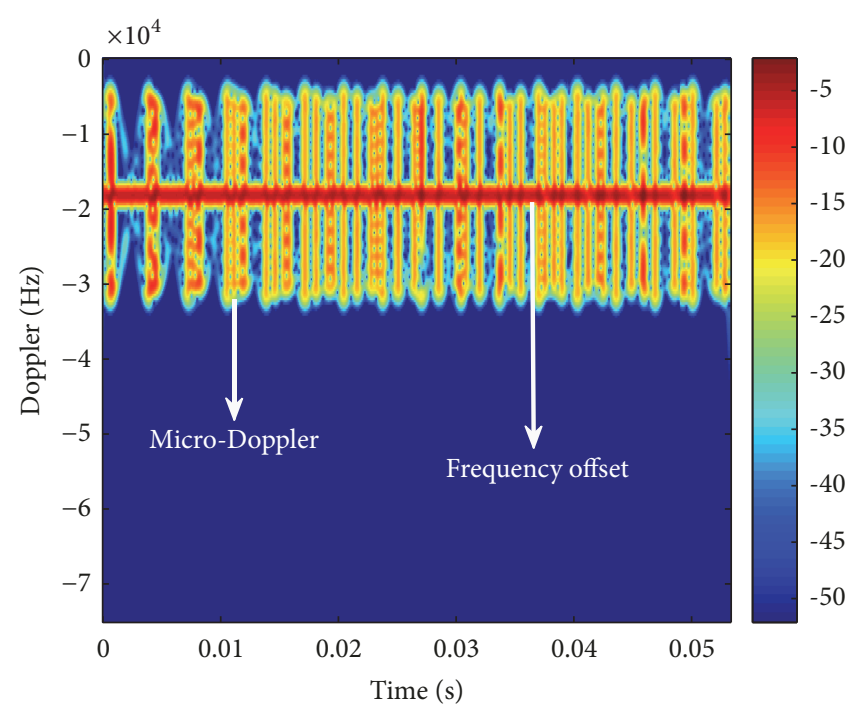

Figure 9: Micro-Doppler of target 1.

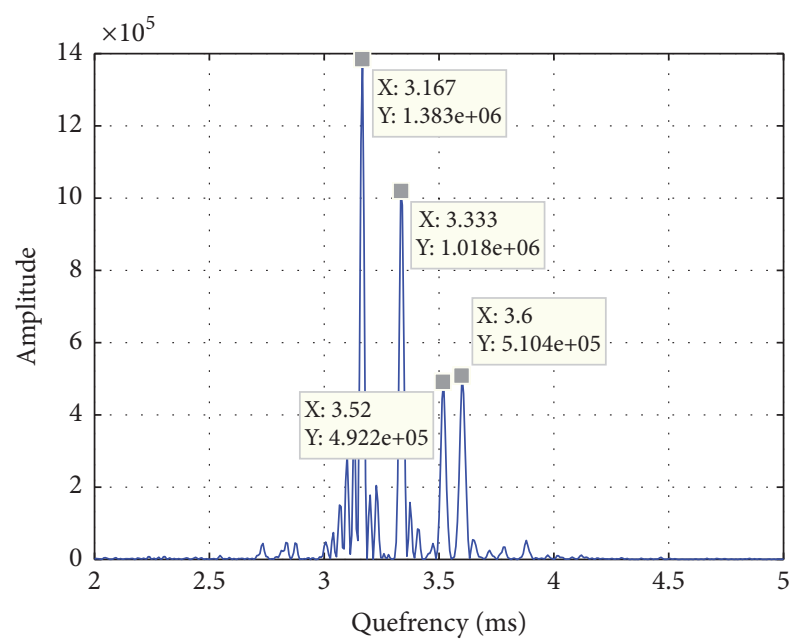

Figure 10: Cepstrum extraction of target 1.

the rotor changes sinusoidally over time. The difference in the amplitude of the micro-Doppler at different moments is due to the different contributions of rotor scattering to echoes at different radar observation angles.

There are multiple rotors in this target; therefore, the micro-Doppler signal is aliased and the type parameters of the UAV rotor cannot be directly obtained. The cepstrum method is used to determine the rotor type.
Figure 10 shows the cepstrum extraction results. It can be seen that target 1 is a UAV with 4 rotors. The rotational speed of the rotors is $157.9 \mathrm{r} / \mathrm{s}, 150.0 \mathrm{r} / \mathrm{s}, 142.0 \mathrm{r} / \mathrm{s}$, and $138.9 \mathrm{r} / \mathrm{s}$. The speed obtained by the cepstrum method is nearly the same as the predetermined speed of the rotors.

In order to verify the robustness of the cepstrum method, noise with different signal-to-noise ratios (SNRs) is added to the echo signal of target 1 . Figure 11 shows the simulation results with $\mathrm{SNR}=-5, \mathrm{SNR}=0$, and $\mathrm{SNR}=5$. It is evident from Figure 11 that after adding the noise, the peak energy of the position corresponding to the rotation period of the rotor is slightly lower. However, there is still a significant difference between the rotor values and the values of other positions and the addition of the noise has no influence on the identification of the number of rotors and the rotational speed.

4.2.2. Identifications of Target 2 and Target 3. Target 2 and target 3 are analyzed as a single target. Figure 12 shows the micro-Doppler characteristics of the two targets.

As shown in Figure 12, there is one rotor for target 2 and target 3. Figure 13 shows that the rotational speed of this rotor obtained by the cepstrum method is $88 \mathrm{r} / \mathrm{s}$.

The position of the single rotor is estimated by the SFM parameter optimization method after the two target body scatterings are removed. The estimated results are shown in Figure 14. The parameters of the three targets in the detection area are shown in Table 2. 


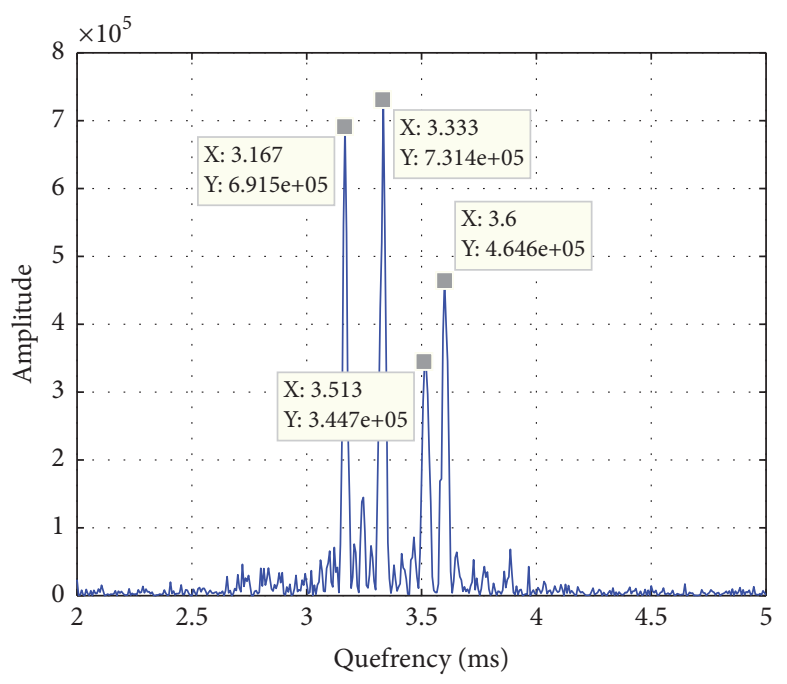

(a) $\mathrm{SNR}=-5 \mathrm{~dB}$

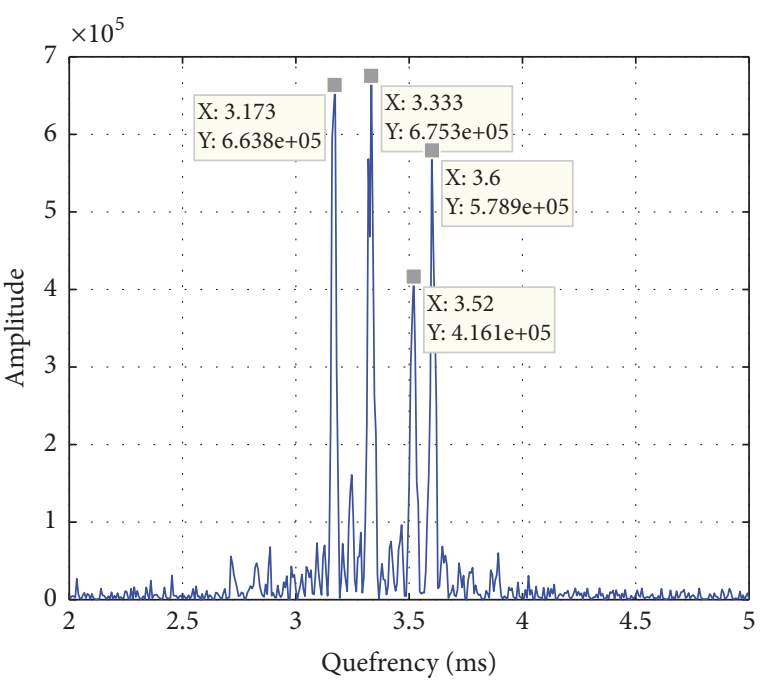

(b) $\mathrm{SNR}=0 \mathrm{~dB}$

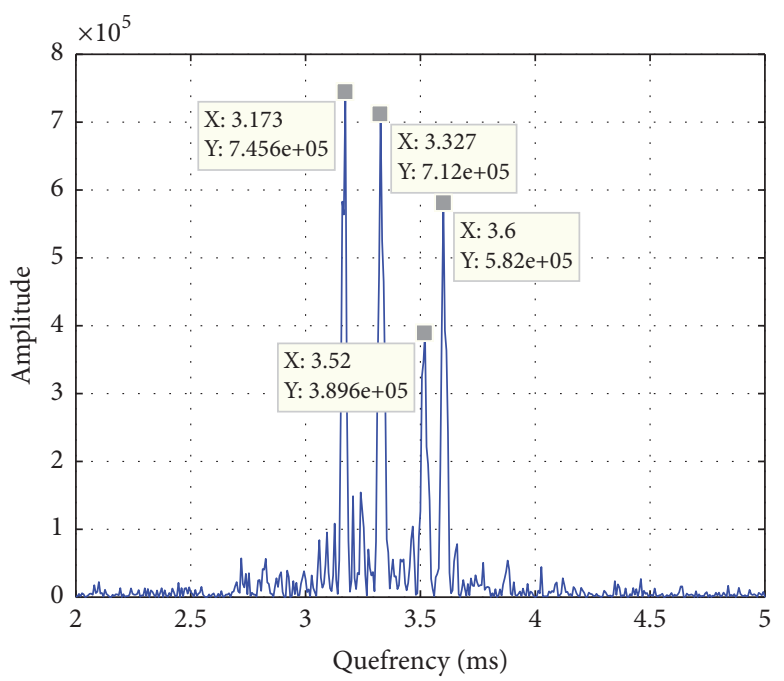

(c) $\mathrm{SNR}=5 \mathrm{~dB}$

FIgURE 11: Cepstrum results with the addition of different SNRs.

Table 2 shows the good accuracy of the detection of the UAV and its position. Figure 15 shows a comparison of the UAVs predetermined route based on the GNSS signal and the actual route based on radar tracking. In the case of a GNSS spoofing attack, the captured UAV has flown away from its predetermined route but the original control source was not alerted because it received data that appeared to be correct from the attackers. In this case, the radar-assisted detection and identification method can be used for real-time monitoring of the UAV independent from the GNSS signal; the deviation between the actual route and predetermined route can be determined and an alert can be sent. It is evident that there is a considerable deviation between the GPS-based positions and the radar positions. The latter method provides accurate positioning of the UAV and allows for effective monitoring of UAVs in the IoT.

\section{Conclusion}

In this paper, a radar-assisted UAV detection and identification method that is independent of a GPS system was proposed. The HRRP technology of wideband radar was used for UAV detection and positioning and the micro-Doppler signal, which is capable of detecting rotating targets, was used for UAV identification. Cepstrum analysis was used to extract the number and rotation speed of the UAV rotors. The simulation results demonstrated the good robustness of this method. An SFM parameter optimization algorithm was used to compensate for the rotational motion and estimate the rotor positions; two UAVs with aliasing signals in the HRRP were separated effectively using the proposed method. The simulation results showed that the UAVs can be identified and the number of UAVs, the number of rotors on each UAV, 


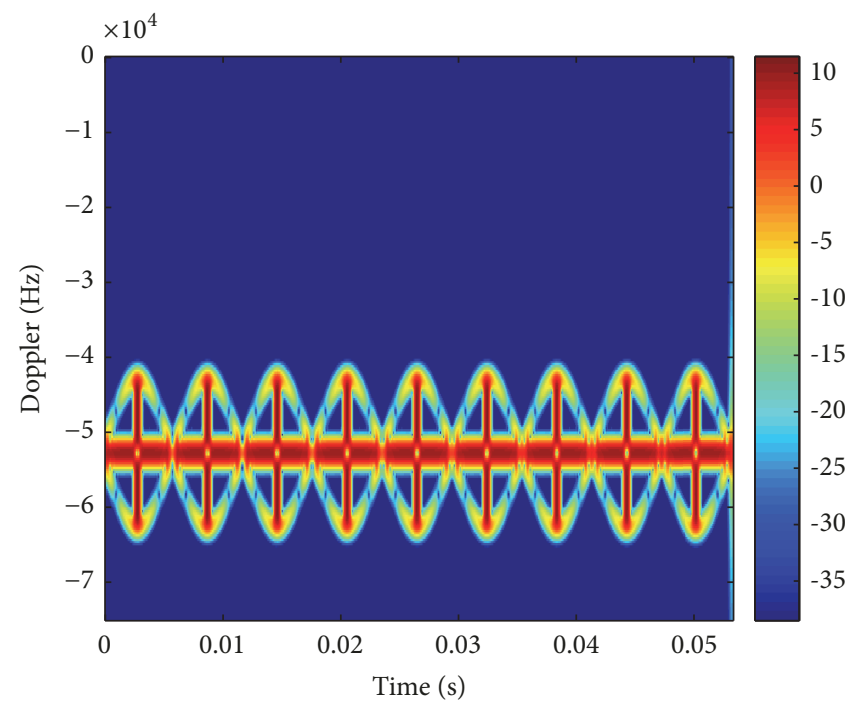

FIgURE 12: Micro-Doppler of target 2 and target 3.

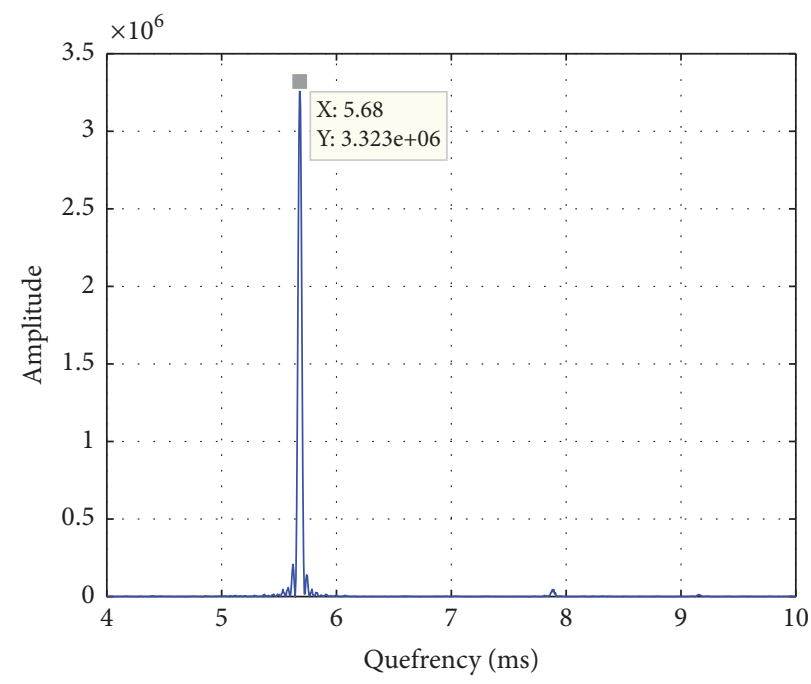

FIGURE 13: Cepstrum result of target 2 and target 3.

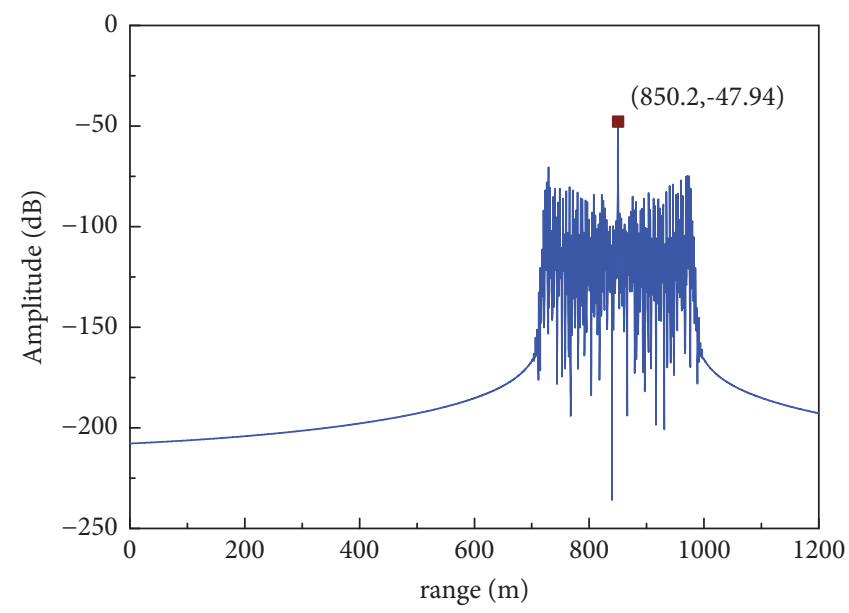

FIGURE 14: Rotor position estimation. 


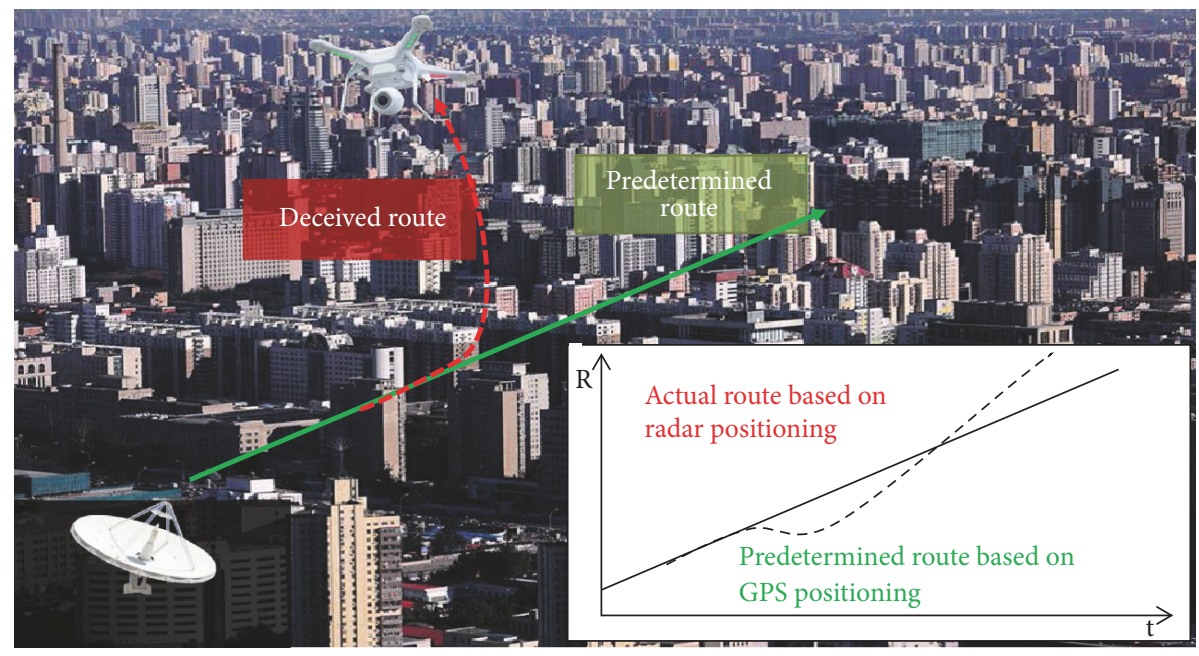

FigURE 15: UAV flight route.

and the rotation speeds of the rotors can be determined. In addition, the proposed radar-assisted UAV detection and identification method can provide alerts if UAVs deviate from their routes during GNSS spoofing attacks.

\section{Data Availability}

The data used to support the findings of this study are available from the corresponding author upon request.

\section{Conflicts of Interest}

The authors declare that there is no conflict of interest regarding the publication of this paper.

\section{References}

[1] A. Fotouhi, M. Ding, and M. Hassan, "Understanding autonomous drone maneuverability for internet of things applications," in Proceedings of the 18th IEEE International Symposium on A World of Wireless, Mobile and Multimedia Networks, WoWMoM 2017, IEEE, China, June 2017.

[2] M. Mozaffari, W. Saad, M. Bennis, and M. Debbah, "Mobile unmanned aerial vehicles (UAVs) for energy-efficient internet of things communications," IEEE Transactions on Wireless Communications, vol. 16, no. 11, pp. 7574-7589, 2017.

[3] A. Giyenko and Y. I. Cho, "Intelligent UAV in smart cities using IoT," in Proceedings of the 16th International Conference on Control, Automation and Systems, ICCAS 2016, pp. 207-210, IEEE, Republic of Korea, 2016.

[4] M. Rieke, T. Foerster, J. Geipel, and T. Prinz, "High-precision positioning and real-time data processing of UAV systems," ISPRS - International Archives of the Photogrammetry, Remote Sensing and Spatial Information Sciences, vol. 38, no. 1, article no. C22, pp. 119-124, 2011.

[5] V. M. Sineglazov and E. V. Daskal, "IEEE 802.15.4/zigbee wireless network as a basis for uav navigation system," Electronics and Control Systems, vol. 2, no. 52, pp. 20-24, 2017.
[6] A. J. Kerns, D. P. Shepard, J. A. Bhatti, and T. E. Humphreys, "Unmanned aircraft capture and control via GPS spoofing," Journal of Field Robotics, vol. 31, no. 4, pp. 617-636, 2014.

[7] V. C. Chen, F. Li, S.-S. Ho, and H. Wechsler, "Micro-doppler effect in radar: Phenomenon, model, and simulation study," IEEE Transactions on Aerospace and Electronic Systems, vol. 42, no. 1, pp. 2-21, 2006.

[8] D. Solomitckii, M. Gapeyenko, V. Semkin, S. Andreev, and Y. Koucheryavy, "Technologies for efficient amateur drone detection in 5G millimeter-wave cellular infrastructure," IEEE Communications Magazine, vol. 56, no. 1, pp. 43-50, 2018.

[9] S. Han, Y. Huang, W. Meng, C. Li, N. Xu, and D. Chen, "Optimal power allocation for scma downlink systems based on maximum capacity," IEEE Transactions on Communications, vol. 67, no. 2, pp. 1480-1489, 2019.

[10] S. Han, Y. Zhang, W. Meng, and H. Chen, "Self-interferencecancelation-based SLNR precoding design for full-duplex relayassisted system," IEEE Transactions on Vehicular Technology, vol. 67, no. 9, pp. 8249-8262, 2018.

[11] J. Martinez, D. Kopyto, M. Schutz, and M. Vossiek, "Convolutional neural network assisted detection and localization of UAV s with a narrowband multi-site radar," in Proceedings of the 2018 IEEE MTT-S International Conference on Microwaves for Intelligent Mobility, ICMIM 2018, IEEE, Germany, April 2018.

[12] Y. Wang, T. Phelps, K. Kibaroglu, M. Sayginer, Q. Ma, and G. M. Rebeiz, "28 GHz 5G-based phased-arrays for UAV detection and automotive traffic-monitoring radars," in Proceedings of the 2018 IEEE/MTT-S International Microwave Symposium - IMS, pp. 895-898, IEEE, Philadelphia, PA, USA, 2018.

[13] S. Sun, M. Kadoch, L. Gong, and B. Rong, "Integrating network function virtualization with SDR and SDN for $4 \mathrm{G} / 5 \mathrm{G}$ networks," IEEE Network, vol. 29, no. 3, pp. 54-59, 2015.

[14] T. Yang, J. Zhao, T. Hong, W. Chen, and X. Fu, "Automatic identification technology of rotor UAVs based on $5 \mathrm{G}$ network architecture," in Proceedings of the 2018 IEEE International Conference on Networking, Architecture and Storage (NAS), pp. 1-9, IEEE, 2018.

[15] S. Han, S. Xu, W. Meng, and C. Li, "An agile confidential transmission strategy combining big data driven cluster and OBF," IEEE Transactions on Vehicular Technology, vol. 66, no. 11, pp. 10259-10270, 2017. 
[16] B. Rong, Y. Qian, K. Lu, H. Chen, and M. Guizani, "Call admission control optimization in WiMAX networks," IEEE Transactions on Vehicular Technology, vol. 57, no. 4, pp. 25092522, 2008

[17] S. Han, S. Xu, W. Meng, and C. Li, "Dense-device-enabled cooperative networks for efficient and secure transmission," IEEE Network, vol. 32, no. 2, pp. 100-106, 2018.

[18] Y. Wu, B. Rong, K. Salehian, and G. Gagnon, "Cloud transmission: a new spectrum-reuse friendly digital terrestrial broadcasting transmission system," IEEE Transactions on Broadcasting, vol. 58, no. 3, pp. 329-337, 2012.

[19] B. R. Mahafza, Radar Systems Analysis and Design Using MATLAB, Chapman and Hall/CRC, 2nd edition, 2005.

[20] V. C. Chen, F. Li, S. S. Ho, and H. Wechsler, "Analysis of micro-doppler signatures," IEEE Proceedings-Radar, Sonar and Navigation, vol. 150, no. 4, pp. 271-276, 2003.

[21] M. Jian, Z. Lu, and V. C. Chen, "Experimental study on radar micro-Doppler signatures of unmanned aerial vehicles," in Proceedings of the 2017 IEEE Radar Conference, RadarConf 2017, pp. 0854-0857, IEEE, USA, May 2017.

[22] G. E. Smith, K. Woodbridge, and C. J. Baker, "Radar microdoppler signature classification using dynamic time warping," IEEE Transactions on Aerospace and Electronic Systems, vol. 46, no. 3, pp. 1078-1096, 2010.

[23] C. Weidong, X. Shanjia, W. Dongjin, and L. Falin, "Range performance analysis in linear FMCW radar," in Proceedings of ICMMT 2000. 2000 2nd International Conference on Microwave and Millimeter Wave Technology Proceedings (Cat. No. 00EX364), pp. 654-657, IEEE, Beijing, China, 2000.

[24] Y. Wang, S. Ji, and H. Xu, "Non-stationary signals processing based on STFT," in Proceedings of the 2007 8th International Conference on Electronic Measurement and Instruments, ICEMI, pp. 3301-3304, IEEE, China, August 2007.

[25] H. K. Kim and R. C. Rose, "Cepstrum-domain acoustic feature compensation based on decomposition of speech and noise for asr in noisy environments," IEEE Transactions on Audio, Speech and Language Processing, vol. 11, no. 5, pp. 435-446, 2003.

[26] S. Furui, "Speaker-independent isolated word recognition using dynamic features of speech spectrum," IEEE Transactions on Signal Processing, vol. 34, no. 1, pp. 52-59, 1986. 


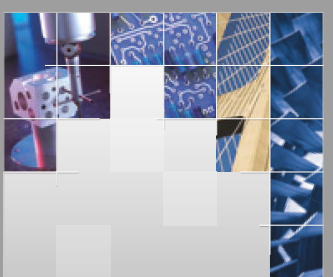

\section{Enfincering}
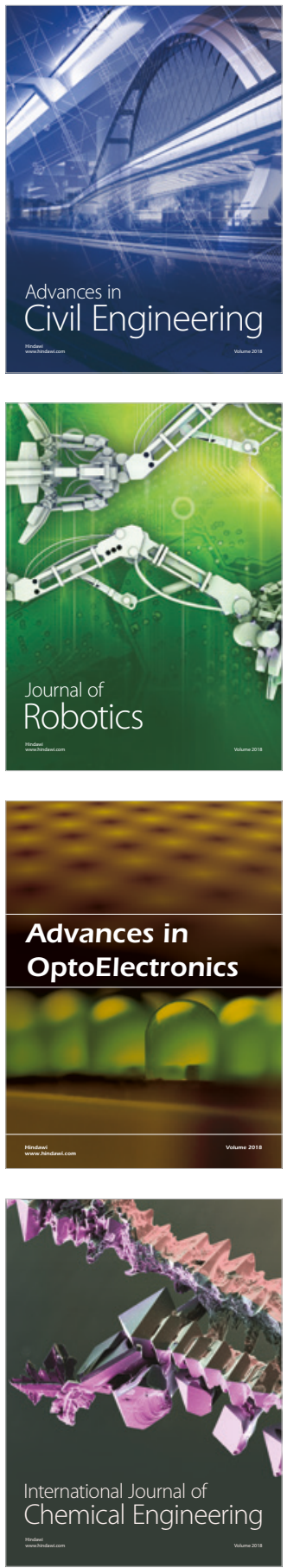

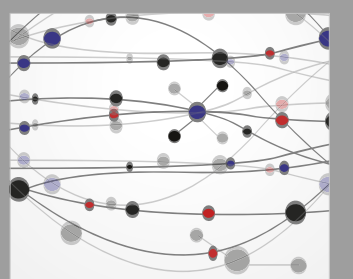

\section{Rotating \\ Machinery}

The Scientific World Journal

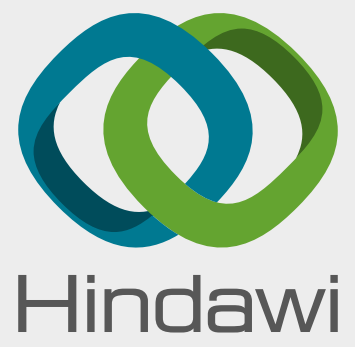

Submit your manuscripts at

www.hindawi.com
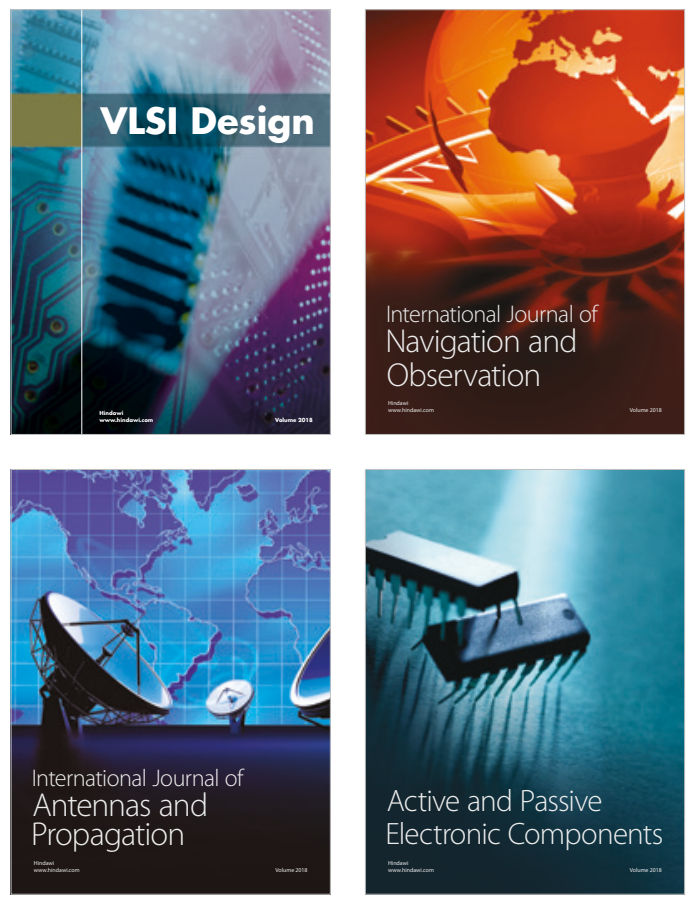
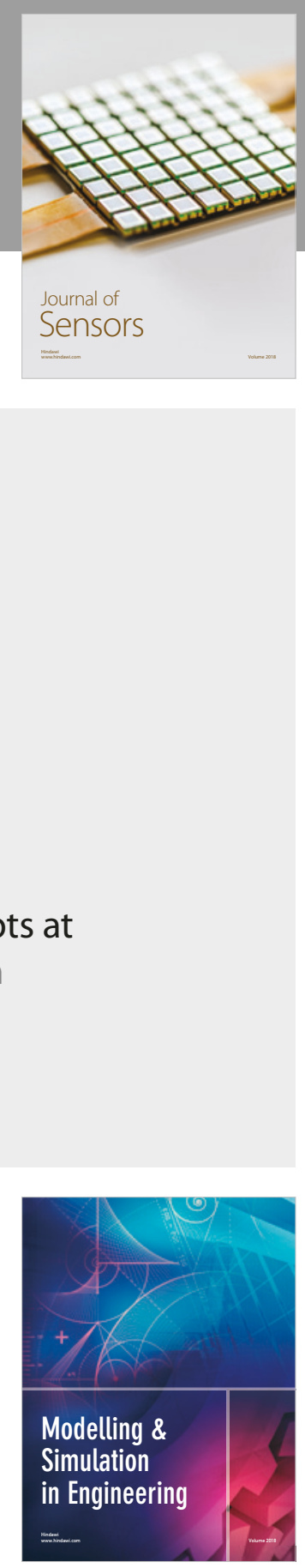

\section{Advances \\ Multimedia}
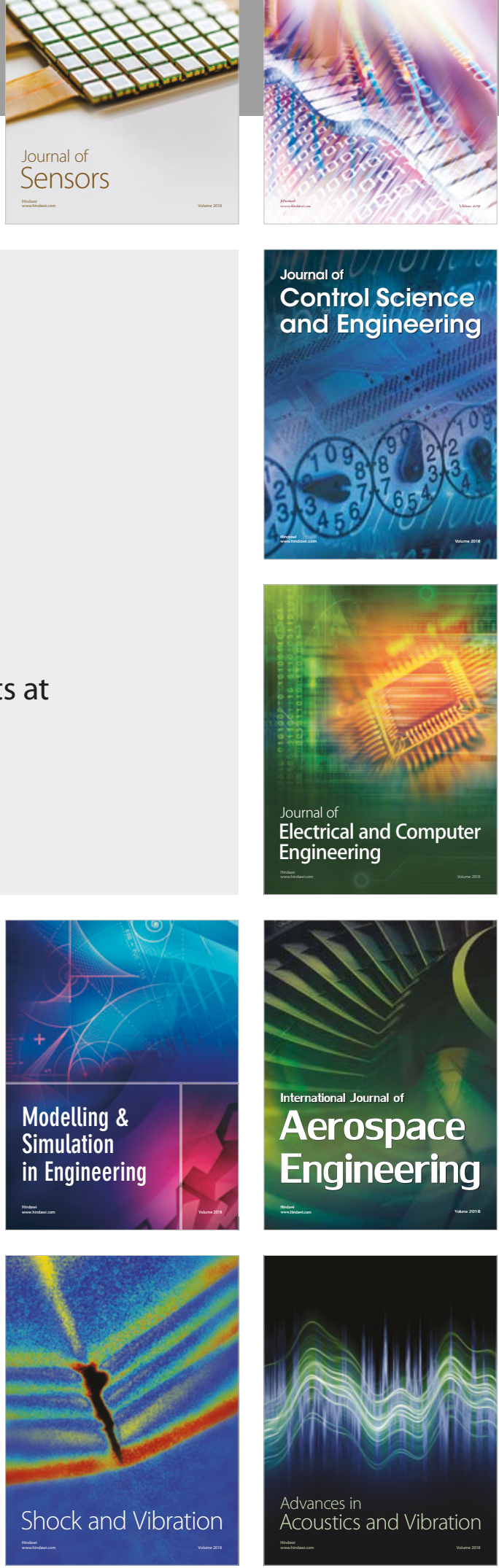